\title{
Fluxo de calor no solo modelado a partir de dados de temperatura em dois níveis de profundidade em uma floresta tropical na Amazônia Ocidental
}

\author{
Soil heat flux modeled from temperature data at two depth levels in a rainforest in Western \\ Amazonia
}

Antônio Vinicius do Prado Rodrigues ${ }^{1}$, Nelma Tavares Dias Soares ${ }^{2}$, Renata Gonçalves Aguiar ${ }^{3}$, Alberto Dresch Webler ${ }^{3}$ e Bruno Soares de Castro ${ }^{4}$

${ }^{1}$ Curso de graduação em Ciências Biológicas, CEULJI/ULBRA, Ji-Paraná, Brasil

${ }^{2}$ Curso de graduação em Engenharia Ambiental, UNIR, Ji-Paraná, Brasil

${ }^{3}$ Departamento de Engenharia Ambiental, UNIR, Ji-Paraná, Brasil

${ }^{4}$ Departamento de Estatística, UNIR, Ji-Paraná, Brasil

\section{Resumo}

O clima global é diretamente dependente do equilibrio ecológico das florestas, sobretudo tropicais. O fluxo de calor no solo é um importante fator em estudos de balanço energético representando a principal forma de troca de energia entre solo e atmosfera. O presente trabalho teve por objetivo estimar ofluxo de calor no solo utilizando medidas de temperatura do solo em dois niveis de profundidade em uma floresta tropical na Amazônia Ocidental, com o intuito de obter-se dados coerentes tanto para utilização dos valores quanto para preenchimento de falhas em banco de dados. Foram utilizados dados de temperatura e fluxo de calor no solo coletados em uma torre micrometeorológica, pertencente à rede de torres do Programa de Grande Escala da Biosfera-Atmosfera na Amazônia, localizada na Reserva Biológica do Jaru. Os dados estimados apresentaram 94\% de concordância com os dados medidos, os dois possuem comportamentos semelhantes o que possibilita o uso em preenchimento de falhas de forma demonstrativa. No entanto há um atraso nos valores do fluxo de calor no solo estimado em relação ao medido, o que interfere no resultado do modelo, suscitando mais estudos para melhorá-lo.

Palavras-chave: Balanço de energia. Fluxo geotérmico. Preenchimento de falhas

\section{Abstract}

The global climate is dependent of ecological balance of forests, especially tropical. The heat flux in the soil is an important factor in studies of energy balance representing the main form of energy exchange between soil and atmosphere. The aim of the present work was to estimate soil heat flux using soil temperature measurements at two depth levels in a tropical forest in the Western Amazon, in order to obtain coherent data for both the use of the values and for the filling of failures in database. Had been used data on temperature and soil heat flux collected in a micrometeorological tower belonging to the towers network of the Large Scale Biosphere-Atmosphere Program in the Amazon, located in the Jaru Biological Reserve. The estimated data presented 94\% agreement with the measured data, the two have similar behaviors that allow the use in filling of failures in a demonstrative way. However, there is a delay in the estimated values of the heat flux in the soil in relation to the measured one, which interferes in the result of the model, provoking more studies to improve it.

Keywords: Energy balance. Geothermal flow. Gap filling 


\section{Introdução}

O bioma Amazônico é um dos mais sistêmicos e complexos do mundo e se estende ao longo de nove países da América do Sul, o que compreende uma área de 6,4 milhões de quilômetros quadrados, sendo que 63\% estão em território brasileiro (PEREIRA et al., 2010). Diante de sua composição física, química e biológica e das relações entre elas, vários fatores estão estritamente ligados à manutenção do equilíbrio nas diversas esferas que compõem esse ecossistema.

Segundo Andrade et al. (2009) as Florestas Tropicais são essenciais ao equilíbrio do clima regional e global, influenciando diretamente na absorção ou emissão de gases, evapotranspiração, balanço energético, entre outros fatores.

Além do balanço energético entre superfície e atmosfera, em uma modelagem meteorológica, o fluxo de calor no solo também se destaca (SANTOS; SILVA; TAVEIROS, 2013). A quantificação desse elemento é de suma importância pois representa as trocas de energia entre solo e atmosfera de um determinado meio, influenciando diretamente no aumento ou redução do calor latente e sensível, o que, consequentemente influencia nas demais variáveis do solo (GALVANI; ESCOBEDO; PEREIRA, 2001). Em florestas, o fluxo de calor no solo pode representar de 30 a 50\% da radiação líquida para a camada de vegetação rasteira (OGEE et al., 2001).

Tratando-se de estudos climatológicos, a região Amazônica apresenta uma grande dificuldade na produção e organização de dados, mesmo com o aumento de sítios de pesquisa e unidades de coletas de informações. O uso desses dados é essencial na compreensão das condições climáticas, previsão do tempo, dinâmica ambiental entre outros fatores que caracterizam o bioma Amazônico. Porém dependendo da série temporal a ser analisada, alguns estudos se tornam inviáveis devido às falhas nos dados (NASCIMENTO et al., 2010).

Sensores de fluxo de calor, normalmente são utilizados para medir os fluxos de calor no solo, esses são instalados a certa profundidade onde é medida a temperatura do solo para se obter os dados do calor armazenado nesta profundidade (SANTOS; SILVA; TAVEIROS, 2013). Além disso, esses sensores são caros o que eleva os custos das pesquisas e sua exposição às intempéries do campo aumenta a vulnerabilidade a danos nesse tipo de equipamento.

Diante disso, o uso de modelagens estatísticas que estimam informações confiáveis de fluxos de calor no solo visando preencher falhas em banco de dados, é essencial para o desenvolvimento de pesquisas que busquem compreender o balanço energético entre solo e atmosfera. Alguns autores fazem uso de métodos que estimam o fluxo de calor no solo a partir de medidas de temperatura, como Kimball e Jackson (1975), Malek (1993) e outros, porém esses tipos de estudos são escassos sobretudo na Região Amazônica. Diante do exposto, este trabalho tem por objetivo estimar o fluxo de calor no solo utilizando médias de temperatura em dois níveis de profundidade em uma floresta tropical na Amazônia Ocidental.

\section{Material e Métodos}

Os dados utilizados neste trabalho foram coletados na Reserva Biológica do Jaru, (10¹1'11,4”S; 6152'29,9' W) localizado no Estado de Rondônia, onde se encontra instalada uma torre micrometeorológica com altura aproximada de 61,5 m, pertencente à rede de torres do Programa de Grande Escala da Biosfera-Atmosfera na Amazônia, LBA (Figura 1). A Reserva Biológica do Jaru (Rebio Jaru), localiza-se cerca de $80 \mathrm{~km}$ ao norte do município de Ji-Paraná, a 120 m do nível do mar (CULF et al., 1997), com temperatura média de $25{ }^{\circ} \mathrm{C}$, e umidade relativa média de 82\% (ANDRADE et al., 2009).

A Rebio Jaru é uma unidade de conservação federal de proteção integral do bioma Amazônico, criada pelo Decreto 83.716, de 11 de julho de 1979 (BRASIL, 1979), sob a tutela do Instituto Chico Mendes de Conservação da Biodiversidade (ICMBio). A reserva está inserida em uma área de floresta tropical primária classificada como Floresta Ombrófila Aberta (CULF et al., 1997).

Foram utilizados dados de temperatura e fluxo de calor no solo, referentes aos meses de janeiro (incompleto), fevereiro e março de 2017 (dias Juliano de 22 a 90), obtidos através do programa LBA. Os sensores Flux plates SH1, Hukseflux, NLD e Soil thermometers IMAG-DLO (MCM101), instalados no sítio experimental da Rebio Jaru, coletam dados de fluxo de calor no solo e temperatura do solo respectivamente, ambos são armazenados em datalogger com frequência de 10 minutos. Posteriormente, foram calculadas as médias de cada variável para obter dados a cada 30 minutos. Esses foram filtrados em laboratório com intuito de extrair somente dados coerentes, sendo corrigidas as eventuais falhas (AGUIAR, 2013).

A base para criação do modelo para estimativa do fluxo de calor no solo foi o da lei de Fourier para transporte de calor (Equação 1).

$$
G=-k \frac{\partial T}{\partial Z}
$$

onde $\mathrm{G}$ é o fluxo de calor no solo $\left(\mathrm{W} \mathrm{m} \mathrm{m}^{-2}\right)$, $\mathrm{k}$ é a condutividade térmica $\left(\mathrm{W} \mathrm{m}^{-1}{ }^{\circ} \mathrm{C}^{-1}\right)$, $\mathrm{T}$ é a diferença de temperatura do solo $\left({ }^{\circ} \mathrm{C}\right)$ e $\mathrm{Z}$ é a profundidade $(\mathrm{cm})$.

Para a determinação da condutividade térmica (calibração do modelo) foram utilizados $70 \%$ dos dados de fluxo de calor no solo a $5 \mathrm{~cm}$ de profundidade e de temperatura do solo a 2 e $10 \mathrm{~cm}$. Ao rearranjar a equação (1), como proposto por Carvalho, 
Souza e Makino (2013) em seu estudo sobre as propriedades térmicas do solo, foram obtidos valores para k a cada 30 minutos, desses foi feita uma média, definida como valor de condutividade térmica.

Para a validação do modelo, G foi estimado a partir dos outros $30 \%$ de dados de temperatura do solo utilizando a mesma equação descrita anteriormente. Por sua vez, para verificar a concordância entre o valor medido e o estimado do fluxo de calor no solo, foi utilizado o índice de concordância proposto por Willmott, Ackleson e Davis (1985) e a raiz do erro quadrático médio (RMSE).

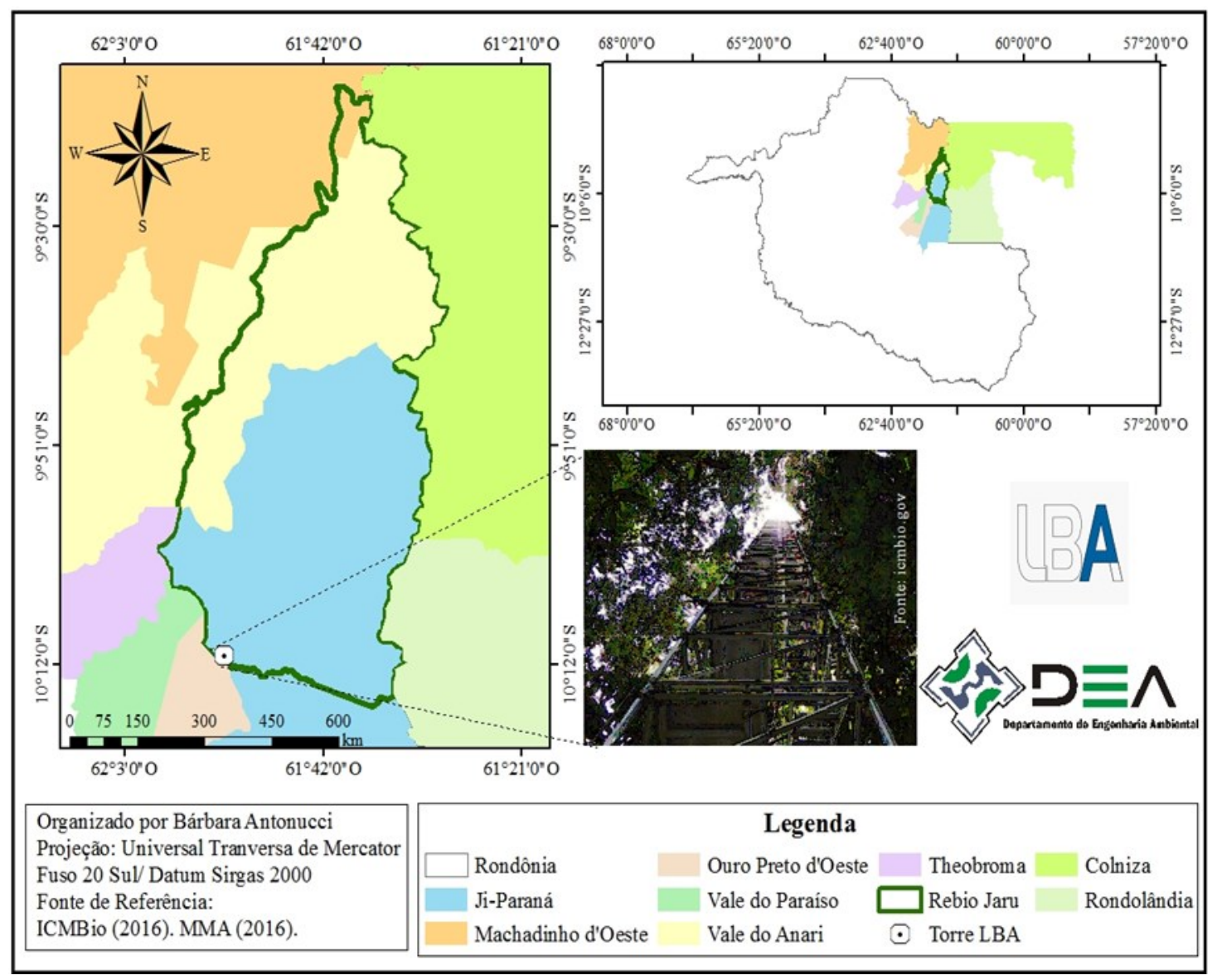

Figura 1 - Localização da área de estudo na Reserva Biológica do Jaru, Rondônia.

Fonte: Antonucci (2016).

\section{Resultados e Discussão}

A condutividade térmica encontrada foi de $0,509 \mathrm{~W} \mathrm{~m}^{-1}{ }^{\circ} \mathrm{C}^{-1}$, esse valor é consistente ao comparar com os valores encontrados por Souza et al. (2006) para uma floresta em Marabá-PA no período úmido. A umidade do solo é um fator importante ao analisar a condutividade, pois a mesma varia principalmente em função do teor de umidade (SOUZA et al., 1996).

O fluxo de calor no solo estimado pelo método ( $\mathrm{G}$ estimado) apresentou um comportamento semelhante ao do fluxo de calor no solo medido pelo sensor ( $G$ medido), com um índice de concordância de aproximadamente 0,94, caracterizando um ótimo desempenho do modelo, pois por mais que haja alguns desvios dos valores predito dos valores reais, o modelo conseguiu capturar de forma satisfatória o comportamento da série (Figura 2). A raiz do erro quadrático médio foi de $1,37 \mathrm{~W} \mathrm{~m}^{-2}$, tal resultado está abaixo do encontrado por Silveira et al. (2013) ao realizar a mesma estimativa $\left(13.28 \mathrm{~W} \mathrm{~m}^{-2}\right)$.

Ao analisar a Figura 3, pode-se observar que o $\mathrm{G}$ estimado condiz em comportamento, mas possui um atraso em relação ao G medido. Provavelmente por esse motivo as médias dos dois tenham se distanciado consideravelmente, sendo a média de $\mathrm{G}$ medido $-0,98 \mathrm{~W} \mathrm{~m}^{-2}$ e a de $\mathrm{G}$ estimado $-0,66 \mathrm{~W} \mathrm{~m}^{-2}$. Quanto ao desvio padrão, foram encontrados $2,92 \mathrm{e} 2,67 \mathrm{~W} \mathrm{~m}^{-2}$ para $\mathrm{G}$ medido e $\mathrm{G}$ estimado, respectivamente.

As estimativas obtidas pelo modelo proposto, quando analisados o índice de concordância e o RMSE, mostraram-se eficientes para preenchimento de falhas em dados demonstrativos, visto que os dados estimados representam o mesmo 


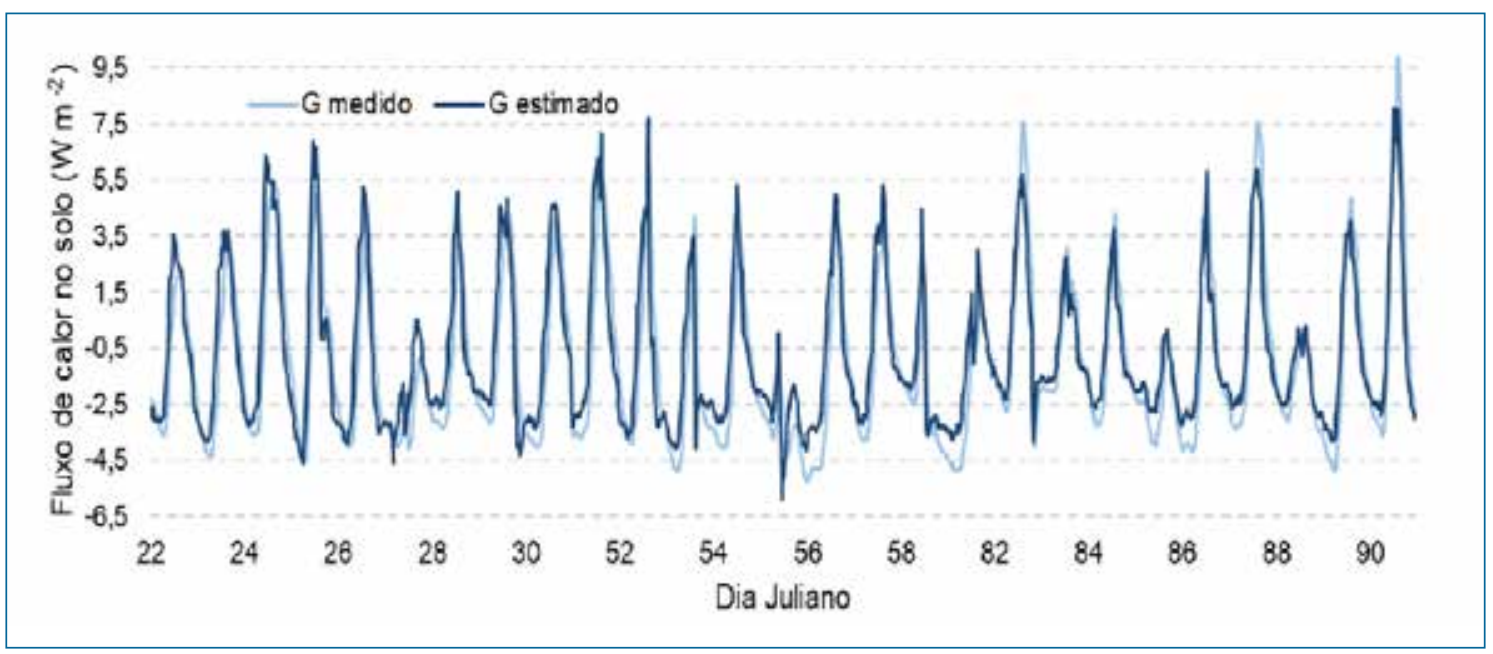

Figura 2 - Média diária do fluxo de calor no solo (G) medido e estimado no ano de 2017, na Reserva Biológica do Jaru, Rondônia

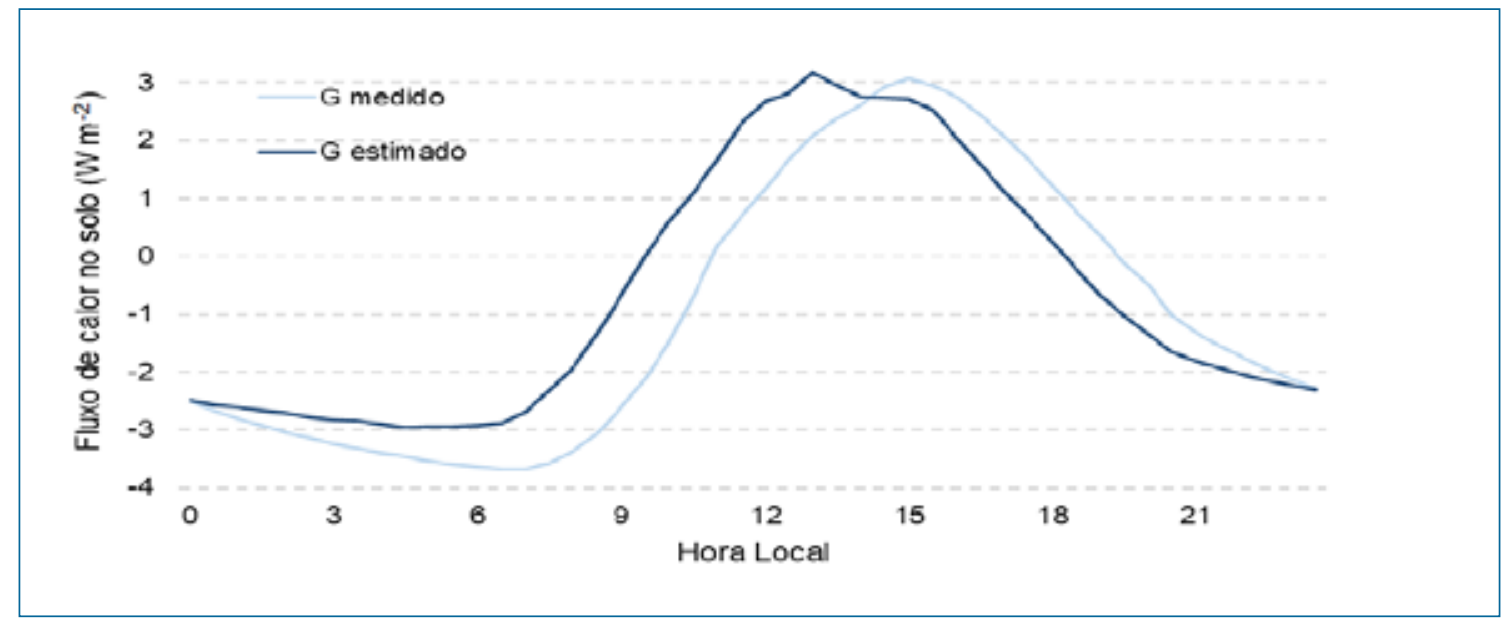

Figura 3 - Média horária do fluxo de calor no solo medido e estimado, na Reserva Biológica do Jaru, Rondônia

comportamento que se tem nos dados medidos. Isso corrobora com os estudos de Baba, Vaz e Costa (2014) que observaram resultados relevantes na utilização de métodos estatísticos e geoestatísticos para identificação de dados inconsistentes e na estimativa de dados a serem preenchidos.

Não obstante, se o atraso observado for ajustado possivelmente representará um ganho expressivo na estimativa do fluxo de calor no solo e seus resultados serão melhores empregados no preenchimento de falhas do banco de dados. Essa será a próxima etapa do presente estudo

\section{Conclusões}

O fluxo de calor no solo em área de floresta tropical pode ter o comportamento bem representado utilizando a lei de Fourier para estimar os valores do mesmo a partir das medidas de temperatura em dois níveis de profundidade, no entanto para que os valores estimados sejam melhores ajustados com os valores medidos é necessário que sejam explicadas as causas do atraso observado para propor uma correção. 


\section{Agradecimentos}

À Fundação de Amparo à Pesquisa de Rondônia (FAPERO), à Coordenação de Aperfeiçoamento de Pessoal de Nível Superior (CAPES), ao Programa de Grande Escala da Biosfera-Atmosfera na Amazônia (LBA), ao Instituto Chico Mendes de Conservação da Biodiversidade (ICMBio) e à Universidade Federal de Rondônia (UNIR), Campus de Ji-Paraná.

\section{Referências}

AGUIAR RG. Balanço de energia em ecossistema Amazônico por modelo de regressão robusta com bootstrap e validação cruzada [thesis]. Cuiabá: Programa de Pós-Graduação em Física Ambiental/ UFMT;2013.

ANDRADE NLR, AGUIAR RG, SANCHES L, ALVES ECRF, NOGUEIRA JS. Partição do saldo de radiação em áreas de floresta amazônica e floresta de transição Amazônia-Cerrado. Rev. Bras. Meteorol. 2009;24(3):346-355.

ANTONUCCI B. Perfil vertical da concentração de CO2 e fluxos de carbono em uma área de floresta na Amazônia Ocidental [monography]. Ji-Paraná: Departamento de Engenharia Ambiental/UNIR; 2016.

BABA RK, VAZ MSMG, COSTA J. Correção de dados agrometeorológicos utilizando métodos estatísticos. Rev. Bras. Meteorol. 2014;29(4):515-526.

LEI N. 83.716 DE 11 DE JULHO DE 1979. Cria no território Federal de Rondônia, a Reserva Biológica do Jaru e dá outras providências. Diário Oficial da União (Brasília). 1979 Jul 12.

CARVALHO SP, SOUZA JRS, MAKINO M. Observações e estimativas de propriedades térmicas do solo sob floresta e pastagem no leste da Amazônia. Rev. Bras. Meteorol. 2013;28(3):331-340.

CULF AD, FISCH G, MALHI Y, NOBRE C A. The influence of the atmospheric boundary layer on carbon dioxide concentrations over a tropical forest. Agric. For. Meteorol. 1997;85:149-158.

GALVANI E, ESCOBEDO JF, PEREIRA AB. Balanço de radiação e fluxo de calor no solo em ambiente natural e protegido cultivado com pepineiro. Bragantia. 2001;60(2):139-147.

KIMBALL BA, JACKSON RD. Soil heat-flux determination: a null-alignment method. Agric. For. Meteorol. 1975;15:1-9.

MALEK E. Rapid changes of the surface soil heat flux and its effects on the estimation of evapo-transpiration. J. Hydrol. 1993;142:89-97.

NASCIMENTO TS, SARAIVA JMB, SENNA R, AGUIAR FEO. Preenchimento de falhas em banco de dados pluviométricos com base em dados do CPC (Climate Prediction Center): estudo de caso do rio SolimõesAmazonas. RBClima. 2009;7:143-158.

OGEE J, LAMAUD E, BRUNET Y, BERBIGIER P, BONNEFOND JMA. Long-term study of soil heat flux under a forest canopy. Agric. For. Meteorol. 2001;106:173-186.

PEREIRA D, SANTOS D, GUIMARÃES MVJ, VERÍSSIMO A. Fatos Florestais da Amazônia. A Amazônia. Belém: Imazon; 2010. p. 18.

SANTOS MAF, SILVA FM, TAVEIROS FEV. Estimativa de fluxo de calor no solo em Natal/RN baseado na derivada temporal fracionária de meia ordem. Sociedade e Território. 2013;25(2):1-16.

SILVEIRA MC, ZIMMER T, MOREIRA VS, ROBERTI DR. Estimativa do fluxo de calor no solo para diferentes profundidades. Ciênc. Nat. 2013;266-269.

SOUZA JRS, MAKINO M, ARAÚJO R LC, COHEN JCP, PINHEIRO FMA. Thermal properties and heat fluxes in soils under Forest and pasture, in Marabá, PA, Brazil. Rev. Bras. Meteorol. 2006;21(3):89-103. 
SOUZA JRS, PINHEIRO FMA, ARAUJO RLC, PINHEIRO JRHS, HODNETT MG. Temperature and Moisture profiles in Soil Beneath Forest and Pasture Areas in Eastern Amazônia. In: Gash, JHC, Nobre CA, Roberts JM, Victoria RL, editors. Amazonian deforestation and climate. Chichester: John Wiley; 1996. p. 125-137.

WILLMOTT CJ, ACKLESON SG, DAVIS RE. Statistics for the evaluation and comparison of models. JGR. 1985;90(C5):8995-9005.

Antônio Vinicius do Prado Rodrigues

Curso de graduação em Ciências Biológicas, CEULJI/ULBRA, Ji-Paraná, Brasil E-mail: vini.ouropreto@gmail.com

Nelma Tavares Dias Soares

Curso de graduação em Engenharia Ambiental, UNIR, Ji-Paraná, Brasil E-mail: ntds08@gmail.com

Renata Gonçalves Aguiar

Departamento de Engenharia Ambiental, UNIR, Ji-Paraná, Brasil E-mail: rgaguiar@unir.br

Alberto Dresch Webler

Departamento de Engenharia Ambiental, UNIR, Ji-Paraná, Brasil E-mail: betowebler@gmail.com

Bruno Soares de Castro

Departamento de Estatística, UNIR, Ji-Paraná, Brasil E-mail: bbruno1988@gmail.com 